\title{
The Effectiveness of Alkaline Water in Hydrating Athletes
}

\author{
Anna Kurylas ${ }^{1}$, Tomasz Zajac ${ }^{2}$, Grzegorz Zydek ${ }^{3}$, *Adam Zajac ${ }^{3}$ \\ ${ }^{1}$ Phd student - Academy of Physical Education im. J. Kukuczki in Katowice, Poland \\ ${ }^{2}$ Human Performance Laboratory, Academy of Physical Education im. J. Kukuczki in Katowice, Poland \\ ${ }^{3}$ Department of Sports Nutrition, , Academy of Physical Education im. J. Kukuczki in Katowice, Poland
}

Received: February 22,2017; Accepted: March 24,2017; Published: April 07, 2017

*Corresponding author: Adam Zajac, Department of Sports Training, Academy of Physical Education im. J. Kukuczki in Katowice, Poland, E-mail: a.zajac@awf.katowice.pl

The most important ingredient of the human body includes water. Water consists $60-65 \%$ of an adult's body mass, $55 \%$ or less for women, while it reaches values of close to $75 \%$ in infants. A proper hydration state is a must for general health, yet it is even more important for individuals that exercise on a regular basis, and especially for competitive athletes submitted to extreme loads, often under extreme environmental conditions [1]. Intra and extra cellular water is responsible for numerous functions in our bodies. Water allows for homeostasis, it facilitates most biochemical reactions, it allows numerous particles and compounds to dilute; it helps in the transport of metabolites and speeds up the utilization of by-products. Water plays a major role in thermoregulation; it nourishes and moisturizes tissues and organs. Effective hydration determines proper functioning of the whole body, at rest and especially during and after exercise. During exercise heat is produced by the muscles and other organs and it must be eliminated, not to cause hyperthermia. Excess heat produced during exercise has to be removed, and it is mostly accomplished through sweating, what causes significant water loss. Reducing water volume by $2 \%$ or more doing exercise has significant consequences on functions of particular organs and the whole body. Dehydration can decrease aerobic capacity, it can increase blood pressure, cause headaches, decrease concentration and speed of reaction, and may also influence cognitive abilities in sport disciplines that require tactical thinking and decision making [2]. Some studies indicate that even a minor hypohydration of $2 \%$ influences a variety of cognitive functions such as vigilance, alertness, perceptual discrimination, arithmetic ability, visuomotor tracking and psychomotor skills significant in many sport disciplines and everyday life activities $[3,2]$.

There are sport disciplines for which fluid intake before, during and after competition is a key factor determining performance, and those that are not much affected by hydration status. Explosive track and field events such as the shot put or high jump are not influenced to a great degree by hydration status, while performance in aerobic endurance events, especially those lasting several hours, performed under extreme environmental conditions (temperature, humidity, altitude), are very much dependent on the volume, quality and timing of fluid intake. Road cycling is an excellent example, where races last for 4-6 hours and can be performed under very hot or cold conditions, with significant radiation and at low or high altitudes.

There is significant evidence to indicate that hypohydartion impairs aerobic endurance performance, and these effects are significantly related to the degree of body water deficit [4]. Fatigue in prolonged aerobic exercise do to hypohydration is explained by thermoregulatory, cardiovascular and metabolic factors. Dehydration in such circumstances increases core temperature and causes elevation of heart rate parallel to the decrease in blood flow, stroke volume, cardiac output and skin blood flow [5]. Since evaporation is the most effective manner of heat loss during exercise, core body temperature rises at a greater extent as hypohydration levels increase. Dehydration during exercise may also affect muscle metabolism by accelerating the rate of glycogen depletion [6].

Hypohydration and its consequences may also occur in sport disciplines that require high intensity and intermittent bouts of effort over several hours of competition or training. This is especially true in soccer tournaments played in high temperature, with insufficient rest and hydration between games. Some authors [7] reported decreased performance when hypohydration acceded $2.4 \%$ in soccer, while no impairments were observed in shooting, passing or spiking in basketball and volleyball when hypohydration reached $2 \%$ [8]. Tennis and especially long 4-5 set matches played in severe heat have shown 
to cause significant hypohydration and negatively affect shot precision and speed of reaction [2].

It is unclear how much hypohydration impairs muscular strength, and inconsistent results may be due to the use of different protocols to achieve hypohydration and measurements of strength and power abilities. Some authors have observed significant reductions in isometric and isokinetic force after $2 \%$ hypohydration [5], while others did not register significant changes in muscular activity even after a $4 \%$ hypohydration [9]. It seems that strength and power as well as aerobic capacity may be significantly affected by a state of hypohydration in combat sports, where athletes usually dehydrate before competition to reduce body mass for a certain weight category, and then sweat immensely during competition. This has been observed in tournaments which last for several hours during a day in judo, wrestling and boxing [10]. The applied power diminishes significantly in the later stages of these bouts. This has been explained by an increase in core temperature which affects the sequence of muscle strength production by reducing motor cortex activation, peripheral stimulus and power output [9]. A dehydration of $5 \%$ causes serious consequences in the cardio respiratory and muscular systems, decreasing aerobic capacity by $20-30 \%$. Hypohydration in excess of $10 \%$ may be dangerous to the athlete's health and a threat to life. An appropriate hydration of the body is crucial during exercise for adaptive changes to occur at all levels of the organism and also very significant after training and competition for recovery.

Athletes engaged in high intensity exercise for 2 hours a day should consume at least 3-4 1 of water or other fluids. Thirst should not be the main factor determining hydration, since it always appears with a certain delay, while fluids should be replaced on the spot, if possible every 10-15 minutes of activity, with an increased intake during recovery [11].

The demand for water in athletes is conditioned by numerous factors, which include volume, intensity and frequency of training sessions, temperature, humidity, environment, preexercise hydration status, diet, supplementation and clothing. Individual metabolic characteristics also must be considered, as some athletes have much higher BMRs with greater thermogenesis. As a consequence they produce more heat and sweat to a greater extent, losing significant amounts of water [12].

Exercising in a hot environment (above 25C) causes significant disturbances in blood hemodynamics. The skin conductivity increases 7 - fold, what stimulates sweating and evaporation. Well trained athletes undergoing intensive exercise sweat in excess of 2-3l/h, while in extreme conditions even up to $5-7 \mathrm{l} / \mathrm{h}$. A daily loss of $10 \mathrm{l}$ of water under conditions of extreme heat and humidity causes a significant loss of electrolytes, enzymes and stimulates hyponatremia. Endurance athletes can continue to exercise with the inner temperature close to or even above 40C, yet without sufficient hydration, fatigue settles in, hindering coordination and sports performance. Prolonged exercise under such conditions can cause irreversible changes in the body and can even be fatal.

This phenomenon has been observed in marathon runners, cyclists, triathletes and soccer players. Water vapor pressure seems an important factor in exercise tolerance, because of its thermoregulatory effect. In such cases water is lost while exhaling, which can increase up to $1500 \mathrm{ml} / \mathrm{d}$ in a cold dry climate.

Currently there are numerous products available on the market used for hydrating athletes before, during and after exercise. The main objective is to increase exercise tolerance and speed up the recovery process. Most sport drinks are fortified with carbohydrates, minerals and vitamins, or chosen amino acids. The most popular drinks are isotonic in nature, while hypotonic and hypertonic drinks are also used depending on the type of activity, its length and environment in which the exercise is performed [12].

Despite the abundance of sports drinks on the market, water is still one of the most often used forms of replacing fluids during and after exercise, especially in individuals who are watching their body mass closely. Because exercise especially that of high intensity causes significant disturbances in acidbase balance, it has been hypothesized that drinking alkaline water could increase the buffering capacity of blood and muscle tissues and improve the hydration status of athletes. Since most biochemical reactions are very sensitive to $\mathrm{pH}$, drinking alkaline water could facilitate most of these reactions and speed up postexercise recovery.

Water, the most widely used fluid during exercise comes in different forms, with specific properties depending on the mineral content. The mineral content, and especially the proportions between S042- and HCO3- as well as the pH determine hydration status and other therapeutic properties. This has been documented in numerous experiments and clinical trials [13]. Mineral water has a significant impact on acid-base balance which determines anaerobic exercise capacity [14]. Many researchers have suggested that water rich in Ca is characterized by this specific quality [15]. Even subtle changes in blood and tissue $\mathrm{pH}$ have significant metabolic consequences, including the response to oxidative stress [16]. During supramaximal exercise, there is a significant increase in Reactive Oxygen Species (ROS) and reactive nitrogen species (RNS). In trained individuals the antioxidant system is more efficient due to the adaptation to 
exercise [11]. The neutralization of ROS may promote the use of water rich in hydrogen ions. This action is explained by the stimulation of numerous antioxidant proteins [1]. The results of long-term research show that the use of hydrogen-rich water is helpful in preventing metabolic diseases, including diabetes [17]. Alkaline mineral water with a high $\mathrm{pH}$, through the effect on acid-base balance can also increase the rate of lactate utilization following anaerobic exercise [18].

Acid-base equilibrium within the body is tightly maintained through the interaction of three complementary mechanisms: Blood and tissue buffering systems (e.g., bicarbonate), the diffusion of carbon dioxide from the blood to the lungs via respiration, and the excretion of hydrogen ions from the blood to urine by the kidneys [19].

Many studies have shown that the consumption of alkalizing supplements can have a significant effect on the body's acid-base balance using surrogate markers of urine and blood $\mathrm{pH}$ [16]. It seems possible that the regular consumption of alkaline water could have a similar effect on markers of acid-base balance, yet the available data is conflicting, thus further research is necessary with different populations, different exercise protocols and varied environmental conditions.

For practical purposes, different methods of evaluating hydration status are used. They include total body water content (electrical impedance), blood variables (osmolality, volume and sodium concentration), as well as urine variables (osmolality, volume, specific urine gravity. Research indicates that most athletes do not meet the daily norms of water intake, considering their training loads, energy demands and sweating rate [11]. Athletes practicing and competing in long distance endurance events are most prone to dehydration, yet hypohydration has also been observed in team sport games and combat sports. An experiment conducted in extreme heat $\left(31^{\circ} \mathrm{C}\right)$ and high humidity (60-65\%) on youth athletes subjected to a continuous run $\left(65 \% \mathrm{VO}_{2 \max }\right)$ until volitional exhaustion showed no difference in hydration status when different types of fluid were ingested during the exercise protocol. Unrestricted intake of tap water, grape-flavored water and grape-flavored water with $6 \%$ carbohydrate and $18.0 \mathrm{mmol} / \mathrm{L} \mathrm{NaCl}$ showed no differences in performance and hydration status after the exercise protocol [4]. Alkaline water with a specific mineral content and increased $\mathrm{pH}$ has recently been proposed as an alternative sports drink. There are reports indicating a positive effect of alkaline water on resting and post-exercise acid-base balance [18]. The results of this study indicates, that the use of water with alkalizing properties exhibits a significant potential for hydration, it reduces fluid-electrolyte disturbances and accelerates the rate of lactate utilization following intensive anaerobic interval exercises. Skeletal muscles fatigue by numerous mechanisms, including accumulation of metabolites, such as potassium, or $\mathrm{H}+$ [11]. To a large extent, the structural damage to the myocytes and inflammation depend on the exercise ROS production [20]. Several authors have indicated that consumption of alkaline water inhibits the production of ROS during exercise of high intensity [20]. A randomized double blind study showed that the consumption of highly alkaline water with a pH of 9,3 allowed for increased resting and post-exercise blood $\mathrm{pH}$ and a lower level of metabolic acidosis after an intensive physical effort [21].

In another randomized study conducted on premenopause women, regular consumption of $1,5 \mathrm{l} / \mathrm{d}$ of highly alkaline water caused a significant increase in bone density, increased urine $\mathrm{pH}$ and a decrease of parathyroid hormone and serum C-telopeptide [22]. Other studies indicate that regular consumption of alkaline water prevents diabetes and atherosclerosis by lowering blood concentration of glucose and HbA1c, as well as Triglycerides (TG), Low Density Lipoproteins (LDL) and Total Cholesterol (TCH). Some authors also indicate that drinking alkaline water may slow down neurodegenerative processes [23].

In competitive sports urine osmolality, specific urine gravity and color are often used as indicators of the state of hydration [24]. Consumption of alkaline water following a dehydrating bout of cycling exercise has previously been shown to rehydrate cyclists faster and more completely than the consumption of placebo water. Following the consumption of alkaline water, the cyclists demonstrated less total urine output, their urine was more concentrated (higher specific gravity), and total blood protein concentration was lower [25]. In another well controlled experiment Heil [26] reported that water retention at the end of a 3-hour recovery period was 79.2 $\pm 3.9 \%$ when subjects drank alkaline water versus $62.5 \pm 5.4 \%$ when consuming a placebo.

It can be concluded that the habitual consumption of highly alkaline mineralized water can significantly improve hydration status. Alkaline water with a $\mathrm{pH}$ as high as 9.3 can improve resting and exercise acid-base balance and thus improve both, aerobic and anaerobic performance. Daily recommendations for alkaline water intake should be strictly individualized, and in case of competitive athletes depend on their age, sex, body mass, BMR, training volume and intensity, diet, type of sport discipline and the environment in which the training or competition takes place. Thus, children and youth athletes with a body mass of 25$35 \mathrm{~kg}$ and low energy expenditure can consume as little as $1 \mathrm{l}$ of water a day, while male adult road cyclists $(70-75 \mathrm{~kg})$ performing a continuous effort of 4-5h in a hot and humid environment may need up to 7-81 of alkaline water to fully hydrate the body, and utilize its full potential for exercise and recovery. 


\section{References}

1. Montain SJ. Hydration recommendations for sport 2008. Curr Sports Med Rep. 2008;7(4):187-192. doi: 10.1249/JSR.0b013e31817f005f.

2. Cian C, Barraud PA, Melin B, Raphel C. Effects of fluid ingestion on cognitive function after heat stress or exercise induced dehydration. Int J Psychophysiol. 2001;42(3):243-251.

3. Cian C, Koulmann PA, Barraud PA, Raphed C, Jimenez C, Melin B. Influence of variations of body hydration on cognitive performance. J Psychophysiol. 2000;14(1):29-36.

4. Wilk B, Timmons BW, Bar-Or O. Voluntary fluid intake, hydration status, and aerobic performance of adolescent athletes in the heat. Appl Physiol Nutr Metab. 2010;35(6):834-841. doi: 10.1139/H10-084.

5. Judelson DA, Maresh CM, Anderson JM, Armstrong LE, Casa DJ, Kraemer WJ, et al. Hydration and muscular performance - Does fluid balance affect strength, power and high-intensity endurance. Sports Med 2007;37(10):907-921.

6. Jentjens RL, Wagenmakers AJ, Jeukendrup AE. Heat stress increases muscle glycogen use but reduces the oxidation of ingested carbohydrates during exercise. J Appl Physiol. 2002;92(4):1562-1572.

7. Edwards AM, Mann ME, Marfell-Jones MJ, Rankin DM, Noakes TD, Shilington DP. Influence of moderate dehydration on soccer performance physiological responses to $45 \mathrm{~min}$ of outdoor match-play and the immediate subsequent performance of sport specific and mental concentration tests. Br J Sports. Med. 2007;41(6):385-391.

8. Hoffman JR, Stavsky H, Falk B. The effect of water restriction on anaerobic power and vertical jumping height in basketball players. Int Sports Med. 1995;16(4):214-218.

9. Hayes LD, Morse CI. The effect of progressive dehydration on strength and power; is there a dose-response? Eur J Appl Physiol. 2009;108(4):701-707. doi: 10.1007/s00421-009-1288-y.

10.Sawka MN. Physiological consequences of hypohydration: exercise performance and thermoregulation. Med Sci Sports. Exercs 1992;24(6):657-670.

11. Maughan RJ, Noakes TD. Fluid replacement and exercise stress. A brief review of studies on fluid replacement and some guidelines for the athlete. Sports Med. 1991;12(1):16-31.

12. Coyle EF. Fluid and fuel intake during exercise. Journal of Sports Sciences. 2004;22(1):39-55.

13. Petraccia L, Liberati G, Giuseppe Masciullo S, Grassi M, Fraioli A. Water, mineral waters and health. ClinNutr. 2006;25(3):377-385.
14. Kawamura T, Huang CS, Peng X, Masutani K, Shigemura N, Billiar TR, Okumura M, Toyoda Y, Nakao A: The effect of donor treatment with hydrogen on lung allograft function in rats. Surgery. 2011;150(2):240249. doi: 10.1016/j.surg.2011.05.019.

15. Kajiyama S, Hasegawa G, Asano M, Hosoda H, Fukui M, Nakamura N, et al. Supplementation of hydrogen-rich water improves lipid and glucose metabolism in patients with type 2 diabetes or impaired glucose tolerance. Nutr Res. 2008;28(3):137-143. doi: 10.1016/j.nutres.2008.01.008.

16. Konig D, Muser K, Dickhuth HH, Berg A, Deibert P. Effect of a supplement rich in alkaline minerals on acid-base balance in humans. Nutr J. 2009;8:23. doi: 10.1186/1475-2891-8-23.

17. Ohta S, Nakao A, Ohno K. The 2011 Medical Molecular Hydrogen Symposium: An inaugural symposium of the journal Medical Gas Research. Med Gas Res. 2011;1(1):10. doi: 10.1186/2045-9912-1-10

18. Chycki J, Zając T, Maszczyk A, Kurylas A. The effect of mineral-based alkaline water on hydration status and the metabolic load during short-term anaerobic exercise. Biology of Sport. 2017;34:255-261. DOI: $10.5114 /$ biolsport.2017.66003

19. Palazzetti S, Rousseau AS, Richard MJ, Favier A, Margaritis I. Antioxidant supplementation preserves antioxidant response in physical training and low antioxidant intake. Br J Nutr. 2004;91(1):91-100.

20. Ostojic SM, Stojanovic MD. Hydrogen-Rich Water Affected Blood Alkalinity in Physically Active Men. Res in Sport Med. 2014;22(1):49-60. doi: 10.1080/15438627.2013.852092.

21.Wynn E, Burckhard P, Lanham-New SA, Krieg MA. Positive influence of nutritional alkalinity on bone health. Proceedings of the Nutrition Society. 2010;69(1):166-173. doi: 10.1017/S002966510999173X

22. Santaka S, Takeki H, Kiichiro T. Advanced research on the health benefit of reduced water. Trends in Food Science and Technology. 2012;23(2):124-131.

23. Oppliger RA, Magnes SA, Popowski LA. Accuracy of urine specific gravity and osmolarity as indicators of hydration status. Int J Sport Nutr Exerc Met. 2005;15(3):236-251.

24. Heil DP, Seifer J. Influence of bottled water on rehydration following a dehydrating bout of cycling exercise. J Int Sports Nut. 2009;6(Suppl 1):9. doi: 10.1186/1550-2783-6-S1-P9

25. Heil DP. Acid-base balance and hydration status following consumption of mineral-based alkaline bottled water. J IntSoc Sports Nutr. 2010;7:29-41. doi: 10.1186/1550-2783-7-29. 